\title{
Perivascular Epithelioid Cell Tumors (PEComas) of the Orbit
}

\author{
Panagiotis Paliogiannis · Giuseppe Palmieri ${ }^{1}$. Francesco Tanda • Antonio Cossu \\ Department of Surgical, Microsurgical and Medical Sciences, University of Sassari, Sassari; \\ ${ }^{1}$ Institute of Biomolecular Chemistry, Cancer Genetics Unit, C.N.R., Sassari, Italy
}

Dear editor,

We read with great interest the case recently reported by Kim et al. ${ }^{1}$ on a rare malignant perivascular epitheliod cell neoplasm (PEComa) involving the right lung. As mentioned by Kim et al. ${ }^{1}$ and as we reported in a previous article, PEComas are very rare, with approximately 50 reported cases in several anatomical sites, including the bladder, uterus, vulva, vagina, prostate, lung, liver, pancreas, and other tissues. ${ }^{2}$ In this letter, we briefly describe our experience with a PEComa in an unusual anatomical site, the orbit.

The lesion involved a 46-year-old male patient who was referred to surgery for a painful round lesion of the internal aspect of the right orbit. The surgeons removed the lesion, which measured $1.5 \mathrm{~cm}$ in maximum diameter and presented a brownish color and a soft-elastic consistency. The lesion was well circumscribed and characterized by an expansive growth pattern. It predominantly comprised epithelioid cells with clear to lightly eosinophilic cytoplasm and occasionally showed granular cell changes. The epithelioid elements were organized in aggregates, trabeculas, and cordons, often surrounding vascular structures (Fig. 1). The nuclei presented a round-shaped, vesicular aspect with frequent nucleoli. The mitotic rate was 2 mitoses per 10 high power fields. No signs of necrosis or vascular invasion were evidenced, and the margins of resection were free of tumor. The immunohistochemical results were as follows: strong positivity for human melanoma black 45 (HMB-45) (Fig. 2) and cathepsin

\section{Corresponding Author}

Panagiotis Paliogiannis, MD, PhD

Department of Surgical, Microsurgical and Medical Sciences, University of Sassari,

Viale San Pietro 43, 07100, Sassari, Italy

Tel: +39079228596, Fax: +39-0079228596,

E-mail: panospaliogiannis@gmail.com

Received: September 29, 2016 Revised: October 21, 2016

Accepted: October 26, 2016
$\mathrm{K}$, weak positivity for actin, and negativity for Melan-A, vimentin, S-100, and cytokeratins. Despite the benign aspect of the lesion and the low mitotic rate, a careful follow-up was suggested,

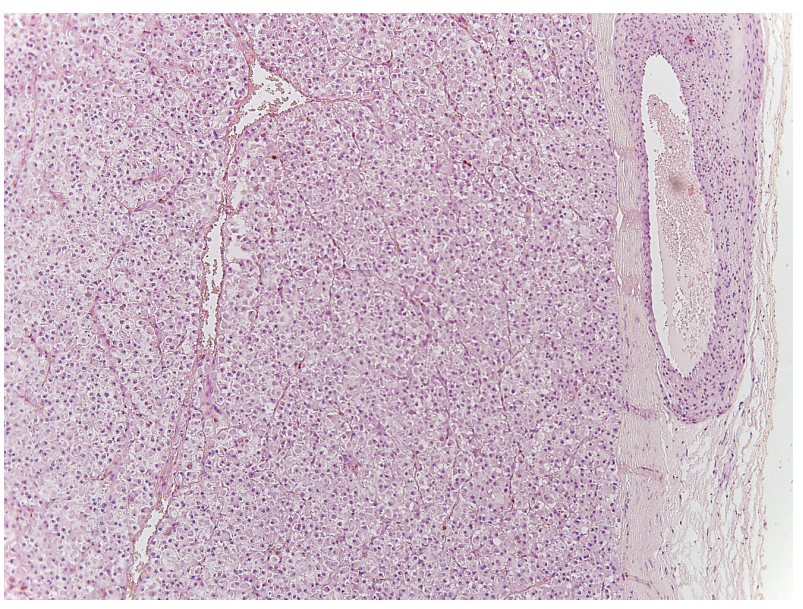

Fig. 1. A section of the lesion stained with hematoxylin and eosin, evidencing its microscopic features and perivascular origin.

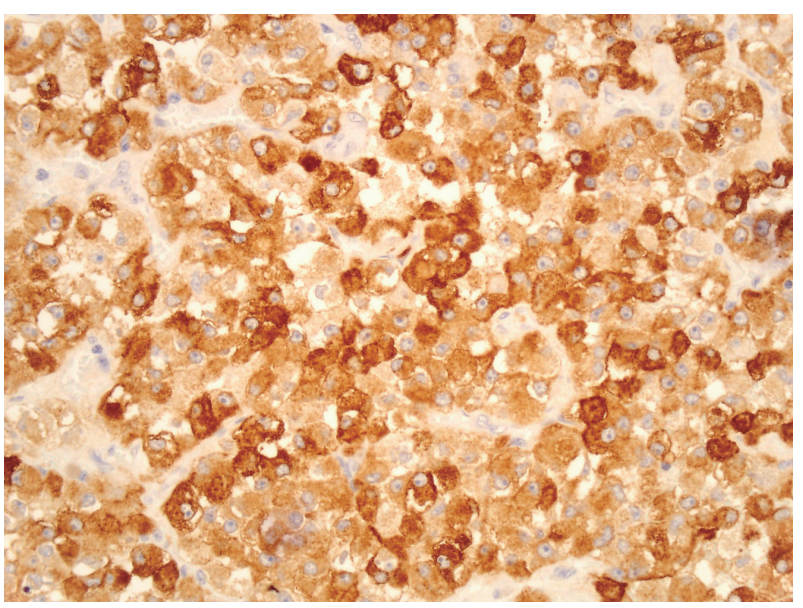

Fig. 2. Human melanoma black 45 immunostaining of the tumor. 
because of the lack of prognostic data due to the small number of cases described in the literature. No recurrence of the disease was observed in the final clinical evaluation 50 months after surgery.

Orbital PEComas are very rare. According to Goto et al., ${ }^{3}$ only four cases of PEComa with ocular involvement have been reported in the recent literature, and only two of them originated from the orbit. The first orbital case was described in 2005 by Iyengar $e t a l .^{4}$ in a 9-year-old female patient, and the authors demonstrated that characteristic histologic features and an immunohistochemical profile of negativity for epithelial markers and positivity for melanogenesis-related markers are useful to define such lesions. The second case was described in 2008 by Guthoff et al. ${ }^{5}$ in a 54-year-old man. In that case, the lesion was surgically removed, and no recurrence has occurred after 17 months of follow-up. The tumor showed strong immunohistochemical positivity for both HMB-45 and Melan-A and a low proliferation index (Ki-67 < 1\%). Interestingly, in our case, Melan- $A$ was negative, as opposed to the findings of Iyenqar $e t a l{ }^{4}$ and Guthoff et al. The low proliferative activity and low mitotic index found in our case suggest that orbital PEComas are generally benign, as those of other anatomical districts. Nevertheless, aggressive PEComas have been described, and this should be considered in cases of orbital PEComas, especially when features of aggressiveness (necrosis, high mitotic rate, great size, infiltrative growth pattern, hypercellularity) are present.

\section{Conflicts of Interest}

No potential conflict of interest relevant to this article was reported.

\section{REFERENCES}

1. Kim HY, Choi JH, Lee HS, Choi YJ, Kim A, Kim HK. Sclerosing perivascular epithelioid cell tumor of the lung: a case report with cytologic findings. J Pathol Transl Med 2016; 50: 238-42.

2. Cossu A, Paliogiannis P, Tanda F, Dessole S, Palmieri G, Capobianco G. Uterine perivascular epithelioid cell neoplasms (PEComas): report of two cases and literature review. Eur J Gynaecol Oncol 2014; 35: 309-12.

3. Goto H, Usui Y, Nagao T. Perivascular epithelioid cell tumor arising from ciliary body treated by local resection. Ocul Oncol Pathol 2015; 1: 88-92.

4. Iyengar P, Deangelis DD, Greenberg M, Taylor G. Perivascular epithelioid cell tumor of the orbit: a case report and review of the literature. Pediatr Dev Pathol 2005; 8: 98-104.

5. Guthoff R, Guthoff T, Mueller-Hermelink HK, Sold-Darseff J, Geissinger E. Perivascular epithelioid cell tumor of the orbit. Arch Ophthalmol 2008; 126: 1009-11. 